\title{
Systems intervention to promote colon cancer screening in safety net settings: protocol for a community-based participatory randomized controlled trial
}

\author{
Aimee S James ${ }^{1 *}$, Veronica Richardson ${ }^{2}$, Jean S Wang ${ }^{3}$, Enola K Proctor ${ }^{4}$ and Graham A Colditz ${ }^{1}$
}

\begin{abstract}
Background: Colorectal cancer is a leading cause of cancer mortality. Screening can be effective but is underutilized. System- or multi-level interventions could be effective at increasing screening, but most have been implemented and evaluated in higher-resource settings such as health maintenance organizations. Given the disparities evident for colorectal cancer and the potential for screening to improve outcomes, there is a need to expand this work to include diverse settings, including those who treat economically disadvantaged patients. This paper describes the study protocol for a trial designed to increase colorectal cancer screening in those 'safety-net' health centers that serve underinsured and uninsured patients. This trial was designed and is being implemented using a community-based participatory approach.
\end{abstract}

Methods/design: We developed a practical clinical cluster-randomized controlled trial. We will recruit 16 community health centers to this trial. This systems-level intervention consists of a menu of evidence-based implementation strategies for increasing colorectal cancer screening. Health centers in the intervention arm then collaborate with the study team to tailor strategies to their own setting in order to maximize fit and acceptability. Data are collected at the organizational level through interviews, and at the provider and patient levels through surveys. Patients complete a survey about their healthcare and screening utilization at baseline, six months, and twelve months.

Outcomes: The primary outcome is colorectal cancer screening by patient self-report, supplemented by a chart-audit in a subsample of patients. Implementation outcomes informed by the Reach, Efficacy/Effectiveness, Adoption, Implementation, and Maintenance (RE-AIM) conceptual framework will be measured at patient, provider, and practice levels.

Discussion: Our study is one of the first to integrate community participatory strategies to a randomized controlled trial in a healthcare setting. The multi-level approach will support the ability of the intervention to affect screening through multiple avenues. The participatory approach will strengthen the chance that implementation strategies will be maintained after study completion and, supports external validity by increasing health center interest and willingness to participate.

Trial registration: NCT01299493

Keywords: Colon cancer, Healthcare disparities, Screening, Randomized controlled trial, Intervention studies, Multi-level intervention, Implementation strategy, Community-based participatory research

\footnotetext{
*Correspondence: jamesai@wudosis.wustl.edu

'Division of Public Health Sciences, Department of Surgery, Washington University School of Medicine, 660 S. Euclid Ave, Campus Box 8100, St. Louis, MO 63110, USA

Full list of author information is available at the end of the article
} 


\section{Background}

Colorectal cancer (CRC) is a leading cause of cancer death among men and women in the United States [1]. Many CRC deaths could be avoided through prevention and early detection activities; early stage CRC is associated with excellent survival [1]. Routine screening for CRC is recommended starting at age 50 years for adults at average-risk; several different screening tests have been found effective and are recommended at varying intervals [2,3]. CRC is unique among cancers, because screening is associated not only with early detection, but with prevention [4]. Cancers can be averted through the identification and removal of precancerous polyps. Yet, colorectal screening is underutilized; at least $40 \%$ of ageeligible U.S. adults are not adherent to current screening guidelines $[5,6]$. This rate is lower than many other cancer screenings.

There are disparities in screening and survival from CRC. Individuals who are lower income, underinsured or uninsured, or are from a racial/ethnic minority are less likely to get screened and more likely to be diagnosed at later stages $[1,7]$. Uninsured patients fare worse than their insured counterparts even when adjusting for stage at diagnosis [7-12]. Given that CRC is a leading cancer diagnosis and disparately experienced, and that effective prevention and early detection tools reduce mortality by one-half but are under-utilized in routine primary care settings [13,14], it is important to investigate the feasibility and effectiveness of strategies to increase screening.

Interventions to increase CRC rates that are focused on individual level factors have had only minimal to moderate success in getting patients screened. While individuallevel strategies can be effective and have promise, systems- or multi-level approaches have the potential to reach a wider swath of the patient population and thus may have more overall impact [15-18]. Such approaches can set the stage to increase the likelihood of patientprovider discussion about CRC screening as well as increase the likelihood that screening is referred and completed. Most interventions have been tested in structured and well-resourced settings [19-22]. In response to a Request for Applications (CA 09-032) from the National Cancer Institute requesting a randomized trial using Community-Based Participatory Research (CBPR) methods, we met with our Colon Cancer Community Advisory Board $(\mathrm{CAB})$ to identify needs and elicit preferences and suggestions for study design and implementation. Our objective in this study is to conduct a practical clinical trial [23-27] to evaluate the viability and effectiveness of evidence-based implementation strategies in community health centers serving populations disparately affected by CRC. To achieve our stated aims, we are developing, implementing, and evaluating a systems-level intervention aimed at increasing CRC screening. The long-term goal is to reduce CRC disparities by developing sustainable and disseminable implementation approaches that will effectively promote informed decisions about CRC screening across a variety of settings, particularly those considered underserved. Our protocol is described below.

\section{Methods}

This delayed start cluster randomized controlled study is designed as a 'practical clinical trial' [23-28]. As such, it is designed to test the impact of an evidence-based intervention under 'real world' conditions. Using a rolling recruitment, community health centers (CHCs) are randomized to either intervention or comparison (i.e., delayed intervention). The primary outcome is CRC screening rates as assessed by patient self-report. Other outcomes, such as implementation fidelity and reach, will be evaluated according to the Reach, Efficacy/Effectiveness, Adoption, Implementation, and Maintenance (RE-AIM) conceptual framework [29-33]. This study underwent review by the Washington University Human Research Protection Office (IRB Protocol \#201110005).

\section{Role of community-based participatory research in this study}

Based on the RFA and the input from our $C A B$, we designed this research to follow established CBPR principles, including acknowledging the community; fostering co-learning and capacity building for all; building on strengths and resources within the community; integrating and achieving a balance of all partners; facilitating collaborative, equitable partnership in all phases of research; a focus on local relevance and determinants of health; involving partners and systems development in a cyclical and iterative process; disseminating finding and knowledge gained to all partners and involving all partners in the dissemination process; and planning for a long-term process and commitment [34,35]. Key aspects of CBPR incorporated in this trial include collaborative partnership during design, development, implementation, and evaluation, an emphasis on local relevance, and a commitment to long-term relationships and sustainability of interventions. As such, the early outcomes and formative work involved in this research inform the procedures used in the latter activities. Partnered activities and decisions occur throughout the trial. Our cancer center's Program for the Elimination of Cancer Disparities had an existing network of community and clinical partners who formed our $\mathrm{CAB}$ and helped facilitate the integration of CBPR into the proposal development and planning.

\section{Theoretical framework}

In addition to applying CBPR principles, another goal of our study was to incorporate a multi-level approach that acknowledged practical factors related to implementa- 
tion across settings. We chose the RE-AIM framework [29-33] as the guide for our measures selection and analysis plan. RE-AIM describes different components of dissemination research and is useful for translating research to practice and for conceptualizing the external validity of a trial. The following RE-AIM concepts are incorporated into our measures and analyses: reach and representativeness, differences in implementation between $\mathrm{CHCs}$, adoption of the trial and of the various evidence-based implementation strategies by $\mathrm{CHCs}$ and providers, effectiveness of the overall intervention in increasing screening, and maintenance of resultant strategy changes after the end of data collection. The study aims to reach patient, healthcare providers, and systems that affect care and care provision.

\section{Setting and participants}

\section{Community health centers}

To be eligible, CHCs must serve mostly Medicaid, uninsured, or lower-income patients; be willing to be randomized to intervention or comparison, and willing to allow the research team access to $\mathrm{CHC}$ managers/directors, patients, and providers. Sites $(n=16)$ are recruited on a rolling basis. We chose this approach in order to better serve the sites' timetables and availability, and to tap on their enthusiasm for improving quality of care at their sites. Recruiting all sites at the beginning of the trial would force some $\mathrm{CHCs}$ to wait before the study started at their site, risking dropout due to administrative turnover or emerging or competing needs of the $\mathrm{CHC}$.

There are over $30 \mathrm{CHCs}$ in our metropolitan region. $\mathrm{CHCs}$ were initially identified from amongst our existing community partners. Next, we created a list of federallyqualified $\mathrm{CHCs}$ and other known CHCs in our targeted region, and approached them to assess interest. Where possible, we start with a known contact; in other sites, the health center Chief Executive Officer or Chief Medical Officer is identified from websites. In all cases, potential sites are emailed an Institutional Review Boardapproved invitation letter and study information sheet. Follow-up is conducted by the study team via email and telephone. In most cases, a combination of email, telephone, and in-person contacts will be made before $\mathrm{CHCs}$ formally agree to participate in the trial. With each $\mathrm{CHC}$, we conduct an organizational assessment upon entry and exit into the survey. Additionally, $\mathrm{CHC}$ providers will answer anonymous surveys at the start/end of the study to assess their awareness and perception of the trial. Organizational and provider surveys will help us evaluate the RE-AIM constructs of reach, adoption, implementation, and maintenance. We will also be able to better characterize the representativeness (external validity) of our participating sites.

\section{Patients}

Although the intervention is delivered at the practicelevel, primary outcomes of CRC screening will be assessed via self-report from patients. Patient surveys will inform effectiveness and implementation (for example, if educational materials at the clinic reached patients).

A random sample of patients will be recruited from each participating $\mathrm{CHC}$ for the survey. Inclusion criteria include: age $\geq 49$, English-speaking, having contact information listed in the medical record, and been seen at the $\mathrm{CHC}$ within the last two years. Patients are recruited by mailed invitation letter from the health center about the study and giving them the opportunity to opt out. Study team members will follow-up with all patients who do not opt out. We will aim to collect baseline data from 100 to 110 respondents per clinic where possible. With 70 to $75 \%$ retention by 12 months, this will result in adequate power to detect intervention effects. We recognize that some sites may have smaller patient populations and that retaining participants over the course of the 12-month study may be harder when working with underserved populations whose contact information may change during the study period. However, restricting our sample to only health centers with large or stable patient populations would limit external validity.

The study team considered using chart reviews as the primary outcome. However, given the strength of support for the validity of self-reported CRC screening [36-39], and the difficulty of chart reviews in low-resource settings where electronic medical records may be harder to search, requiring chart reviews would be a barrier to $\mathrm{CHC}$ participation and potentially decrease external validity. We will use a chart audit on a subset of sites for further verification of self-report in this population (see section entitled Data collection - Patient-level data).

\section{Intervention}

The intervention consists of a menu of evidence-based strategies for increasing CRC screening. There are several evidence-based systems interventions to promote CRC screening in primary care, but few [20] have been tested in underserved populations or in real-world settings. We selected the primary strategies that have evidence for their effectiveness based on the CDC Community Guide to Preventive Services [40,41] and the American Cancer Society/ National Colorectal Cancer Roundtable Toolkit for physicians [42,43]: routine patient reminders, provider reminders, provider feedback, and structural changes. These changes can reach the multiple levels of patient care including systems, providers, and patients. $\mathrm{CHCs}$ randomized to the intervention arm will be presented with the standard 'menu' of implementation strategies and will be able to select the strategies they wish to implement for increasing screening. We will partner with individual sites 
to tailor these strategies into specific interventions that are compatible with their site and perceived as offering advantage over current practices. Comparison $\mathrm{CHCs}$ will be offered the menu of strategies at the end of the trial after data collection has ended. All sites receive access to patient education materials.

We chose this 'menu' approach for several reasons: data indicate that healthcare providers are more likely to adhere to an intervention if they helped design and select it [44]; this approach allows sites to decide which strategies are feasible, relevant, and sustainable in their context (e.g., provider feedback might be difficult in a $\mathrm{CHC}$ without electronic records or with basic electronic systems; patient reminders are challenging if patient contact information is not reliable); offering choice enhances generalizability and representativeness by encouraging more wide-spread participation and buy-in by $\mathrm{CHCs}$; and important to our CBPR approach, our community and clinical partners strongly felt that $\mathrm{CHCs}$ need to have an active role in selecting the intervention, rather than being 'told what to do' by the researchers.

Once decision makers and stakeholders at a $\mathrm{HC}$ are identified, the study team will meet with them to discuss their site's challenges (and current practices) regarding CRC screening, and discuss the general main implementation strategies (reminders, feedback, etc.). Through discussion and consensus, the research team will then develop a menu tailored to each site that is then presented back to the same group for their final decisions. Additional personnel from the $\mathrm{CHC}$ will be consulted as needed (e.g., Information Technology officers if a strategy involves the electronic medical record; health center managers if strategies are directed at patients, etc.).

\section{Data collection}

Our primary outcome will be CRC screening (percent of patients up-to-date on CRC screening per the U.S. Preventive Services Guidelines [45]) based on a survey of patients age-eligible for screening at baseline, six months, and twelve months. As we will describe, data are also collected at the provider- and practice-level.

\section{Patient-level data}

The baseline survey will include: demographics, healthcare utilization, medical home, cancer screening, literacy [46], behavior intentions, barriers to CRC screening [47-49], and knowledge about colon cancer. Standard measures from national surveys are used as available. The sixmonth and 12-month surveys include self-reported CRC screening, healthcare utilization, and awareness of screening or educational efforts. CHCs may add site-specific questions if they wish.

\section{Recruitment for the patient survey}

Procedures for patient recruitment will be finalized in collaboration with the participating sites. At most sites, the research team will work with the $\mathrm{CHC}$ to randomly select eligible patients and mail letters of invitation to potential participants. Letters will have an opt-out option and will be followed up by telephone. We expect that some sites will not want to mail letters out due to privacy concerns. In those cases, participants will be recruited in person at the participating health center. We will track the type of recruitment used and evaluate any potential impact or differences.

\section{General procedures for follow-up data collection}

Follow-up data will be collected via telephone survey. These call attempts will be made on multiple days at different times of the day. After approximately five call attempts with no answer, or two voicemail messages, the study staff will mail a letter stating that we are attempting contact. About a week after the letter is mailed, we will attempt one more 'round' of calls. Calling will continue until attempts to reach the participant have been exhausted (determination that there are no working telephone numbers), participants have completed the survey or declined participation, or the participant is more than three weeks past their survey due-date.

\section{Chart review}

Chart review procedures will be worked out with each $\mathrm{CHC}$, who can opt in or out of this review. We aim to recruit at least 10 of our $16 \mathrm{CHCs}$ to participate in the chart review, recognizing that such a procedure is onerous for $\mathrm{CHCs}$ without a flexible electronic medical record system. Chart review data will be used to supplement and support the self-report findings. We aim to review 74 charts per $\mathrm{CHC}$.

\section{Provider-level data}

To understand how the implementation strategies (and the study) are perceived by the people who work at the health center and to assess whether strategies directed at providers actually reached them, we will also conduct brief, anonymous surveys of health center employees. Surveys will be distributed at the start of the study and postintervention. In general, these questions will address RE-AIM principles, including implementation, perceived maintenance, feasibility, acceptability, but we may add questions to the post-intervention survey based on experiences and feedback during implementation. To maximize response, this survey will be brief (one page) and can be mailed in a pre-paid envelope, or faxed to a secure fax machine. For example, if a $\mathrm{CHC}$ chose to develop provider reminders in their electronic record, the post-survey 
would ask if the provider had seen the reminder and what they thought about it.

\section{Practice-level data}

We will conduct an organizational assessment by interviewing clinic administrators in order to address reach, implementation, and maintenance of the intervention and implementation strategies. Interviews will be audiotaped and transcribed, when the interviewee consents. This semi-structured interview will cover: patient characteristics, current efforts to promote CRC screening if any, current CRC screening rates, current efforts to promote other preventive care or disease management, perceived need for change, preferred changes, and barriers to implementation. The research team will work with the $\mathrm{CHC}$ data manager, if necessary, to access some of this information. We will ask our initial contact at the health center to recommend other persons with whom we should speak, such as other level managers, chief operating officers, medical directors, quality improvement personnel, or opinion leaders within the practice. Data, when presented, will be de-identified (both to individual and to health center). Any characteristics that could be used to identify the administrator or the health center/health system will be masked or aggregated. Data will be used to inform the intervention development, but may be analyzed later (e.g., to look at characteristics of $\mathrm{CHCs}$ that did or did not change screening rates). We will aim to conduct at least two interviews per participating site.

\section{Retention of health centers and survey respondents}

We will use multiple evidence-based methods to maximize retention. We do not anticipate site-level attrition once a $\mathrm{CHC}$ is enrolled, because $\mathrm{CHCs}$ are entitled to pick and choose which strategies they wish to implement and the data collection burden on them (organizational assessment and exit interview) is designed to be minimal. However, the participatory process can be burdensome and time-consuming, or perceived burden could deter sites from participation. Our procedures are designed to balance fidelity to CBPR with reducing 'what we ask' from participating sites. $\mathrm{CHCs}$ are reimbursed financially for all phases of the study, though this does not truly capture the time they spend in a participatory approach.

Attrition among patient participants is a potential limitation. We will apply best practices for retention [50-53] and collect complete contact information at enrollment (name, postal address. and at least two telephone numbers). Participants will be asked to list a secondary contact person as a locator. Differential attrition in the patient survey by study arm is unlikely because the intervention is at the center-level and sites are randomized.

We expect some attrition amongst health center employees, more so due to turnover within $\mathrm{CHCs}$ than because of the study. Our post-survey will ask how long the respondent has been at the health center, but because these surveys are not identifiable and not linked, we will not know whether the same people answered a staff survey at pre and post evaluation.

\section{Outcomes and analysis}

Our analysis will examine rates of being up-to-date on CRC screening, accounting for the clustered nature of the data. We will also assess which $\mathrm{CHCs}$ were reached by the study (and how representative they are of area $\mathrm{CHCs}$ ), reach of the intervention within a $\mathrm{CHC}$ (who was 'touched' by the intervention, which strategies were adopted, implementation of strategies, and maintenance of the strategies by analysis of our organizational assessments, staff surveys, and qualitative feedback.

\section{Power}

This study was powered based on patient self-report of screening. With16 CHCs ( $\mathrm{n}=74$ participants per cluster) and intra-class correlation at 0.04 , we will have $80 \%$ power to detect a $15 \%$ point difference in screening between intervention and comparison. Prevalence rates are estimated based on our experiences with similar patient populations, local BRFSS data, and published screening rates. Intervention effects were estimated from the rough average of published percent increases in screening for our potential strategies. These strategies resulted in a range of 12 to 18 percentage point differences [54-64]. If intra-class correlation is higher but the impact is greater, we still maintain at least $80 \%$ power with this sample.

\section{Analysis}

Descriptive analysis will be used to characterize reach, external validity, adoption of strategies, and fidelity to implementation. Effectiveness of the intervention in increasing CRC screening will focus on self-reported patient completion of CRC screening at 12 months post-baseline. Statistical analysis will quantify the intervention effect at the cluster ( $\mathrm{CHC}$ ) and individual (patient) levels. For the individual-level analysis, we will use a generalized linear mixed model, adjusting for individual-level covariates, and cluster-level covariates, and accounting for the clustered nature of the data. Confounders will be identified as those variables that might influence the outcome and their association will be tested in a bivariate model. Variables that are significantly associated with the outcome will be included as potential covariates in the final adjusted model.

\section{Trial status}

The trial is currently ongoing. The first set of health centers have been recruited and patient data collection has begun. 


\section{Discussion}

Increasingly, researchers are trying to balance randomized controlled research with community-based research aimed at decreasing disparities. Our research design is aimed at testing the effect of an implementation intervention to increase colorectal cancer screening, and at the same time assess the viability (and challenge) of translating evidence-based strategies into diverse settings. The implementation of interventions at the practice-level should mean that patients at the $\mathrm{CHC}$ have equal chance to be exposed to an intervention if and when they come for an appointment; other approaches that require patient uptake might disproportionately reach select groups of patients.

Our approach to health center recruitment will allow us to examine whether settings are representative, including examining reach and adoption (two key elements of the RE-AIM theoretical framework) by tracking how many settings were approached, how many participated, and reasons for non-participation [65-68]. The assessment of participation and reasons for non-participation is a critical advancement toward scientific rigor.

Our decision for allowing a 'menu' of implementation strategies balances the ability to test intervention effects with a respect for the different contexts and preferences of each health center. We recognize that this approach is both a strength and a challenge in the current study. The challenge in our 'menu' design is that it will be difficult to know which strategy had impact, because $\mathrm{CHCs}$ can choose as few or as many as they want. However, our main question is whether the intervention approach of offering a menu of strategies is effective. Identifying the specific effect size of each individual strategy is secondary to the question of whether the overall approach can increase screening. We will carefully track organizational characteristics as well as which strategies are adopted and implemented by which CHCs. We plan to explore the differential effectiveness of strategies if there is enough variation. For these reasons, the menu approach strengthens our study and better informs both the literature and prevention practice.

Efforts at reducing disparities and increasing use of appropriate and recommended cancer screening must address the varying contexts in which primary care occurs and the many barriers to screening at the patient, provider, and clinic levels. Practical trials that address external validity, and strive to understand how interventions may (or may not) work in real-world settings are critical to reducing and ultimately eliminating disparities in cancer screening and survival [65-68].

Abbreviations

CRC: Colorectal cancer; CHC: Community health center.

\section{Competing interests}

The authors declared that they have no competing interest.

\section{Authors' contributions}

ASJ conceived the study and its design and led the writing of the manuscript. VR provided insight into developing studies at CHCs and procedures for working with CHCs. JW contributed to the design and selection of outcome measurements. EP advised on the design of the study, the theoretical framework, and the selection of implementation measures. GAC helped conceive the study and helped draft the manuscript. GAC and AJ helped obtained funding. All authors helped revise the manuscript and reviewed the final version. All authors read and approved the final manuscript.

\section{Acknowledgements}

This study is supported by a Community Networks Program Center grant from the National Cancer Institute (U54CA153460). Dr. Colditz is the lead PI for the Center; Dr. James leads the Research Program and this trial. Dr. Enola Proctor and the Dissemination and Implementation Core provided input on the design. The Dissemination Core was supported by the Washington University Institute of Clinical and Translational Sciences grant UL1 TR000448 from the National Center for Advancing Translational Sciences (NCATS) of the National Institutes of Health $(\mathrm{NIH})$. The content is solely the responsibility of the authors and does not necessarily represent the official view of the $\mathrm{NIH}$. We thank Ms. Victoria Anwuri, Program Manager, Program for the Elimination of Cancer Disparities, who provided invaluable grant preparation assistance. We also thank members of our community partnerships, and participating clinics, whose input made this project a success.

\section{Author details}

${ }^{1}$ Division of Public Health Sciences, Department of Surgery, Washington University School of Medicine, 660 S. Euclid Ave, Campus Box 8100, St. Louis, MO 63110, USA. ${ }^{2}$ Grace Hill Health Centers, Quality Improvement and Corporate Compliance, 1717 Biddle Street, St. Louis, MO 63106, USA. ${ }^{3}$ Division of Gastroenterology, Department of Medicine, Washington University School of Medicine, 660 S. Euclid Avenue, Campus Box 8124, Saint Louis, MO 63110, USA. ${ }^{4}$ Brown School, Washington University, St. Louis, MO 63110, USA.

Received: 13 March 2013 Accepted: 29 May 2013

Published: 3 June 2013

\section{References}

1. ACS: Cancer Facts and Figures, 2011. Atlanta: American Cancer Society; 2011.

2. Levin B, Lieberman DA, McFarland B, Andrews KS, Brooks D, Bond J, Dash C, Giardiello FM, Glick S, Johnson D, et al: Screening and surveillance for the early detection of colorectal cancer and adenomatous polyps, 2008: a joint guideline from the american cancer society, the US multi-society task force on colorectal cancer, and the american college of radiology. Gastroenterology 2008, 134(5):1570-1595.

3. U.S. Preventive Services Task Force: Screening for colorectal cancer: U.S preventive services task force recommendation statement. Ann Intern Med 2008, 149(9):627-637.

4. Towler B, Irwig L, Glasziou P, Kewenter J, Weller D, Silagy C: A systematic review of the effects of screening for colorectal cancer using the faecal occult blood test, hemoccult. BMJ 1998, 317(7158):559-565.

5. Joseph DA, King JB, Miller JW, Richardson LC: Prevalence of colorectal cancer screening among adults-behavioral risk factor surveillance system, United States, 2010. MMWR Morb Mortal Wkly Rep 2012, 61(Suppl):51-56.

6. Cancer screening - United States, 2010. MMWR Morb Mortal Wkly Rep 2012, 61(3):41-45.

7. Espey DK, Wu X-CW, Swan J, Wiggins C, Jim M, Ward E, Wingo PA, Howe $H L$, Ries LAG, Miller B, et al: Annual report to the nation on the status of cancer, 1975-2004, featuring cancer in American Indians and Alaska Natives. Cancer 2007, 9999(9999):NA.

8. Le H, Ziogas A, Lipkin SM, Zell JA: Effects of socioeconomic status and treatment disparities in colorectal cancer survival. Cancer Epidemiol Biomarkers Prev 2008, 17(8):1950-1962.

9. Du XL, Fang S, Vernon SW, El-Serag H, Shih YT, Davila J, Rasmus ML: Racial disparities and socioeconomic status in association with survival in a large population-based cohort of elderly patients with colon cancer. Cancer 2007, 110(3):660-669. 
10. Roetzheim RG, Pal N, Gonzalez EC, Ferrante JM, Van Durme DJ, Krischer JP: Effects of health insurance and race on colorectal cancer treatments and outcomes. Am J Public Health 2000, 90(11):1746-1754.

11. Roetzheim RG, Pal N, Tennant C, Voti L, Ayanian JZ, Schwabe A, Krischer JP: Effects of health insurance and race on early detection of cancer. J Natl Cancer Inst 1999, 91(16):1409-1415.

12. Brewster DH, Thomson CS, Hole DJ, Black RJ, Stroner PL, Gillis C: Relation between socioeconomic status and tumor stage in patients with breast, colorectal, ovarian, and lung cancer: results from four national, population based studies. BMJ 2001, 322:830-831

13. Frazier A, Colditz G, Fuchs C, Kuntz K: Cost-effectiveness of screening for colorectal cancer in the general population. J Am Med Assoc 2000, 284(15):1954-1961.

14. Vogelaar I, van Ballegooijen M, Schrag D, Boer R, Winawer SJ, Habbema JD, Zauber AG: How much can current interventions reduce colorectal cancer mortality in the U.S.? Mortality projections for scenarios of risk-factor modification, screening, and treatment. Cancer 2006, 107(7):1624-1633.

15. Clauser SB, Taplin SH, Foster MK, Fagan P, Kaluzny AD: Multilevel intervention research: lessons learned and pathways forward. J Natl Cancer Inst Monogr 2012, 2012(44):127-133.

16. Taplin SH, Anhang Price R, Edwards HM, Foster MK, Breslau ES, Chollette V, Prabhu Das I, Clauser SB, Fennell ML, Zapka J: Introduction: understanding and influencing multilevel factors across the cancer care continuum. J Natl Cancer Inst Monogr 2012, 2012(44):2-10.

17. Anhang Price R, Zapka J, Edwards H, Taplin SH: Organizational factors and the cancer screening process. J Natl Cancer Inst Monogr 2010, 2010(40):38-57.

18. Ayanian JZ, Sequist TD, Zaslavsky AM, Johannes RS: Physician reminders to promote surveillance colonoscopy for colorectal adenomas: a randomized controlled trial. J Gen Intern Med 2008, 23(6):762-767.

19. Wei EK, Ryan CT, Dietrich AJ, Colditz GA: Improving colorectal cancer screening by targeting office systems in primary care practices: disseminating research results into clinical practice. Arch Intern Med 2005, 165(6):661-666.

20. Dietrich AJ, Tobin JN, Cassells A, Robinson CM, Greene MA, Sox CH, Beach $\mathrm{ML}$, DuHamel KN, Younge RG: Telephone care management to improve cancer screening among low-income women: a randomized, controlled trial. Ann Intern Med 2006, 144(8):563-571.

21. Dietrich AJ, Tobin JN, Cassells A, Robinson CM, Reh M, Romero KA, Flood $A B$, Beach ML: Translation of an efficacious cancer-screening intervention to women enrolled in a Medicaid managed care organization. Ann Fam Med 2007, 5(4):320-327.

22. Ganz PA, Farmer MM, Belman M, Malin JL, Bastani R, Kahn KL, Dietrich A, Fielding J: Improving colorectal cancer screening rates in a managed care health plan: recruitment of provider organizations for a randomized effectiveness trial. Cancer Epidemiol Biomarkers Prev 2003, 12(9):824-829.

23. Tunis SR, Stryer DB, Clancy CM: Practical clinical trials: increasing the value of clinical research for decision making in clinical and health policy. JAMA 2003, 290(12):1624-1632.

24. Glasgow RE, Davidson KW, Dobkin PL, Ockene J, Spring B: Practical behavioral trials to advance evidence-based behavioral medicine. Ann Behav Med 2006, 31(1):5-13.

25. Glasgow RE, Magid DJ, Beck A, Ritzwoller D, Estabrooks PA: Practical clinical trials for translating research to practice: design and measurement recommendations. Med Care 2005, 43(6):551-557.

26. Glasgow RE, Nutting PA, King DK, Nelson CC, Cutter G, Gaglio B, Rahm AK, Whitesides $\mathrm{H}$, Amthauer $\mathrm{H}$ : A practical randomized trial to improve diabetes care. J Gen Intern Med 2004, 19(12):1167-1174.

27. Glasgow RE: Practical, practice, and policy relevant trials. Curr Diab Rep 2004, 4(2):111-112

28. D'Agostino RB Sr: The delayed-start study design. N Engl J Med 2009, 361(13):1304-1306.

29. Glasgow RE, Klesges LM, Dzewaltowski DA, Estabrooks PA, Vogt TM: Evaluating the impact of health promotion programs: using the RE-AIM framework to form summary measures for decision making involving complex issues. Health Educ Res 2006, 21(5):688-694.

30. Glasgow RE, Nelson CC, Strycker LA, King DK: Using RE-AIM metrics to evaluate diabetes self-management support interventions. Am J Prev Med 2006, 30(1):67-73.

31. Dzewaltowski DA, Glasgow RE, Klesges LM, Estabrooks PA, Brock E: RE-AIM: evidence-based standards and a Web resource to improve translation of research into practice. Ann Behav Med 2004, 28(2):75-80.
32. Glasgow RE, McKay HG, Piette JD, Reynolds KD: The RE-AIM framework for evaluating interventions: what can it tell us about approaches to chronic illness management? Patient Educ Couns 2001, 44(2):119-127.

33. Glasgow RE, Vogt TM, Boles SM: Evaluating the public health impact of health promotion interventions: the RE-AIM framework. Am J Public Health 1999, 89(9):1322-1327.

34. Minkler M, Wallerstein N: Community-Based Participatory Research for Health. San Francisco: John Wiley \& Sons, Inc; 2002.

35. Israel BA, Schulz AJ, Parker EA, Becker AB: Community-based participatory research: policy recommendations for promoting a partnership approach in health research. Education for Health (Abingdon) 2001, 14(2):182-197.

36. Jones RM, Mongin SJ, Lazovich D, Church TR, Yeazel MW: Validity of four self-reported colorectal cancer screening modalities in a general population: differences over time and by intervention assignment. Cancer Epidemiol Biomarkers Prev 2008, 17(4):777-784.

37. Partin MR, Grill J, Noorbaloochi S, Powell AA, Burgess DJ, Vernon SW, Halek K, Griffin JM, van Ryn M, Fisher DA: Validation of self-reported colorectal cancer screening behavior from a mixed-mode survey of veterans. Cancer Epidemiol Biomarkers Prev 2008, 17(4):768-776.

38. Vernon SW, Tiro JA, Vojvodic RW, Coan S, Diamond PM, Greisinger A Fernandez ME: Reliability and validity of a questionnaire to measure colorectal cancer screening behaviors: does mode of survey administration matter? Cancer Epidemiol Biomarkers Prev 2008, 17(4):758-767.

39. Baier M, Calong N, Cutter G, McClatchey M, Schoentgen S, Hines S, Marcus AC, Ahnen D: Validity of self-reported colorectal cancer screening behavior. Cancer Epidemiol Biomarkers Prev 2000, 9(2):229-232.

40. Sabatino SA, Lawrence B, Elder R, Mercer SL, Wilson KM, DeVinney B, Melillo S, Carvalho M, Taplin S, Bastani R, et al: Effectiveness of interventions to increase screening for breast, cervical, and colorectal cancers: nine updated systematic reviews for the guide to community preventive services. Am J Prev Med 2012, 43(1):97-118.

41. Briss PA, Zaza S, Pappaioanou M, Fielding J, Wright-De Aguero L, Truman BI, Hopkins DP, Mullen PD, Thompson RS, Woolf SH, et al: Developing an evidence-based guide to community preventive services-methods. The task force on community preventive services. Am J Prev Med 2000, 18(1 Suppl):35-43.

42. Levin B, Smith RA, Feldman GE, Colditz GA, Fletcher RH, Nadel M, Rothenberger DA, Schroy PS 3rd, Vernon SW, Wender R: Promoting early detection tests for colorectal carcinoma and adenomatous polyps: a framework for action: the strategic plan of the national colorectal cancer roundtable. Cancer 2002, 95(8):1618-1628.

43. Safarty M, Peterson K, Wender R: How to Increase Colorectal Cancer Screening Rates in Practice: A Primary Care Clinician's Evidence-Based Toolbox and Guide. 2008. http://www.cancer.org/acs/groups/content/documents/document/ acspc-024588.pdf.

44. Pinto RM, Yu G, Spector AY, Gorroochurn P, McCarty D: Substance abuse treatment providers' involvement in research is associated with willingness to use findings in practice. J Subst Abuse Treat 2010, 39(2):188-194.

45. Whitlock EP, Lin JS, Liles E, Beil TL, Fu R: Screening for colorectal cancer: a targeted, updated systematic review for the U.S. preventive services task force. Ann Intern Med 2008, 149(9):638-658.

46. Morris NS, MacLean CD, Chew LD, Littenberg B: The single item literacy screener: evaluation of a brief instrument to identify limited reading ability. BMC Fam Pract 2006, 7:21.

47. Greiner KA, James AS, Born W, Hall S, Engelman KK, Okuyemi KS, Ahluwalia JS: Predictors of fecal occult blood test (FOBT) completion among lowincome adults. Prev Med 2005, 41(2):676-684

48. James AS, Hall S, Greiner KA, Buckles D, Born WK, Ahluwalia JS: The impact of socioeconomic status on perceived barriers to colorectal cancer testing. Am J Health Promot 2008, 23(2):97-100.

49. Greiner KA, Born W, Nollen N, Ahluwalia JS: Knowledge and perceptions of colorectal cancer screening among urban African Americans. J Gen Intern Med 2005, 20(11):977-983.

50. Foy R, Parry J, Duggan A, Delaney B, Wilson S, Lewin-Van Der Broek NT, Lassen A, Vickers L, Myres P: How evidence based are recruitment strategies to randomized trials in primary care? Evidence from seven studies. Fam Pract 2003, 20(1):83-92.

51. Neaton JD, Grimm RH Jr, Cutler JA: Recruitment of participants for the multiple risk factor intervention trial (MRFIT). Control Clin Trials 1987, 8(4 Suppl):41S-53S. 
52. Dennis BP, Neese JB: Recruitment and retention of African American elders into community based research: lessons learned. Arch Psychiatr Nurs 2000, XIV(1):3-11.

53. Ribisl K, Walton M, Mowbray C, Luke D, Davidson W, Bootsmiller B: Minimizing participant attrition in panel studies through the use of effective retention and tracking strategies: review and recommendation. Eval Program Plann 1996, 19(1):1-25.

54. Task Force on Community Preventive Services: Recommendations for client- and provider-directed interventions to increase breast, cervical, and colorectal cancer screening. Am J Prev Med 2008, 35(1, Supplement 1):S21-S25.

55. Mold JW, Peterson KA: Primary care practice-based research networks: working at the interface between research and quality improvement. Ann Fam Med 2005, 3(Suppl 1):S12-S20.

56. Sabatino SA, Habarta N, Baron RC, Coates RJ, Rimer BK, Kerner J, Coughlin SS, Kalra GP, Chattopadhyay S: Interventions to increase recommendation and delivery of screening for breast, cervical, and colorectal cancers by healthcare providers: systematic reviews of provider assessment and feedback and provider incentives. Am J Prev Med 2008, 35(1, Supplement 1):S67-S74.

57. Myers RE, Turner B, Weinberg D, Hyslop T, Hauck WW, Brigham T, Rothermel T, Grana J, Schlackman N: Impact of a physician-oriented intervention on follow-up in colorectal cancer screening. Prev Med 2004, 38(4):375-381.

58. Denberg TD, Coombes JM, Byers TE, Marcus AC, Feinberg LE, Steiner JF, Ahnen DJ: Effect of a mailed brochure on appointment-keeping for screening colonoscopy: a randomized trial. Ann Intern Med 2006, 145(12):895-900

59. Shankaran V, McKoy JM, Dandade N, Nonzee N, Tigue CA, Bennett CL, Denberg TD: Costs and cost-effectiveness of a low-intensity patientdirected intervention to promote colorectal cancer screening. J Clin Oncol 2007, 25(33):5248-5253.

60. Baron RC, Rimer BK, Breslow RA, Coates RJ, Kerner J, Melillo S, Habarta N, Kalra GP, Chattopadhyay S, Wilson KM, et al: Client-directed interventions to increase community demand for breast, cervical, and colorectal cancer screening: a systematic review. Am J Prev Med 2008, 35(1, Supplement 1):S34-S55

61. Ling BS, Schoen RE, Trauth JM, Wahed AS, Eury T, Simak DM, Solano FX, Weissfeld JL: Physicians encouraging colorectal screening: a randomized controlled trial of enhanced office and patient management on compliance with colorectal cancer screening. Arch Intern Med 2009, 169(1):47-55.

62. Dietrich AJ, Carney PA, Winchell CW, Sox CH, Reed SC: An office systems approach to cancer prevention in primary care. Cancer Pract 1997, 5(6):375-381.

63. Dietrich AJ, Tobin JN, Sox CH, Cassels AN, Negron F, Younge RG, Demby $N A$, Tosteson TD: Cancer early-detection services in community health centers for the underserved. A randomized controlled trial. Arch Fam Med 1998, 7(4):320-327. discussion 328.

64. McPhee SJ, Detmer WM: Office-based interventions to improve delivery of cancer prevention services by primary care physicians. Cancer 1993, 72:1100-1112.

65. Glasgow RE: RE-AIMing research for application: ways to improve evidence for family medicine. J Am Board Fam Med 2006, 19(1):11-19.

66. Dzewaltowski DA, Estabrooks PA, Glasgow RE: The future of physical activity behavior change research: what is needed to improve translation of research into health promotion practice? Exerc Sport Sci Rev 2004, 32(2):57-63.

67. Bull SS, Gillette C, Glasgow RE, Estabrooks P: Work site health promotion research: to what extent can we generalize the results and what is needed to translate research to practice? Health Educ Behav 2003, 30(5):537-549,

68. Glasgow RE, Bull SS, Gillette C, Klesges LM, Dzewaltowski DA: Behavior change intervention research in healthcare settings: a review of recent reports with emphasis on external validity. Am J Prev Med 2002, 23(1):62-69.

doi:10.1186/1748-5908-8-58

Cite this article as: James et al:: Systems intervention to promote colon cancer screening in safety net settings: protocol for a community-based participatory randomized controlled trial. Implementation Science 2013 8:58

\section{Submit your next manuscript to BioMed Central and take full advantage of:}

- Convenient online submission

- Thorough peer review

- No space constraints or color figure charges

- Immediate publication on acceptance

- Inclusion in PubMed, CAS, Scopus and Google Scholar

- Research which is freely available for redistribution 